\title{
Neutron skin thickness in the droplet model with surface width dependence: Indications of softness of the nuclear symmetry energy
}

\author{
M. Warda, ${ }^{1,2, *}$ X. Viñas, ${ }^{1, \dagger}$ X. Roca-Maza, ${ }^{1, \ddagger}$ and M. Centelles ${ }^{1, \S}$ \\ ${ }^{1}$ Departament d'Estructura i Constituents de la Matèria and Institut de Ciències del Cosmos, Facultat de Física, Universitat de Barcelona, \\ Diagonal 647, 08028 Barcelona, Spain \\ ${ }^{2}$ Katedra Fizyki Teoretycznej, Uniwersytet Marii Curie-Skłodowskiej, ul. Radziszewskiego 10, 20-031 Lublin, Poland
}

(Received 4 June 2009; revised manuscript received 21 July 2009; published 28 August 2009)

\begin{abstract}
We analyze the neutron skin thickness in finite nuclei with the droplet model and effective nuclear interactions. The ratio of the bulk symmetry energy $J$ to the so-called surface stiffness coefficient $Q$ has in the droplet model a prominent role in driving the size of neutron skins. We present a correlation between the density derivative of the nuclear symmetry energy at saturation and the $J / Q$ ratio. We emphasize the role of the surface widths of the neutron and proton density profiles in the calculation of the neutron skin thickness when one uses realistic mean-field effective interactions. Next, taking as experimental baseline the neutron skin sizes measured in 26 antiprotonic atoms along the mass table, we explore constraints arising from neutron skins on the value of the $J / Q$ ratio. The results favor a relatively soft symmetry energy at subsaturation densities. Our predictions are compared with the recent constraints derived from other experimental observables. Though the various extractions predict different ranges of values, one finds a narrow window $L \sim 45-75 \mathrm{MeV}$ for the coefficient $L$ that characterizes the density derivative of the symmetry energy that is compatible with all the different empirical indications.
\end{abstract}

DOI: $10.1103 /$ PhysRevC.80.024316

PACS number(s): 21.60.-n, 21.65.Ef, 21.10.Gv, 36.10.-k

\section{INTRODUCTION}

Neutron skin thickness is the name in common usage to refer to the difference between the root-mean-square (rms) radii of the neutron and proton density distributions of atomic nuclei:

$$
\Delta R_{n p}=\left\langle r^{2}\right\rangle_{n}^{1 / 2}-\left\langle r^{2}\right\rangle_{p}^{1 / 2} .
$$

Experimentally, the value of the proton rms radius $\left\langle r^{2}\right\rangle_{p}^{1 / 2}$ is obtained from the charge radius. The latter has been measured by electron-nucleus elastic scattering with high accuracy (often the accuracy in charge radii is better than $1 \%$ [1]). In contrast, our knowledge of the neutron distribution in nuclei and of its rms radius $\left\langle r^{2}\right\rangle_{n}^{1 / 2}$, as well as our knowledge of $\Delta R_{n p}$, is to date less precise. This situation looks inadequate in anticipation of the next generation of rare ion accelerator facilities that are planned for the synthesis and study of exotic nuclei, as in RIKEN centers (Japan), in FAIR at GSI (Germany), in the CSR at HIRFL (China), or in FRIB at MSU (U.S.A.). Without securing sufficient knowledge of the neutron distribution in stable nuclei, the prospects of nuclear structure theory in this thriving domain may be compromised. It is expected that parity-violating electron scattering will provide in the nearby future a leap forward in the quest for high-precision determinations of the neutron radius in heavy nuclei [2].

The calibration of the neutron skin thickness of nuclei also is one of the problems at the forefront of nuclear structure by reason of being intimately correlated with the nuclear symmetry energy. Indeed, the symmetry energy is a fundamental quantity

\footnotetext{
*warda@kft.umcs.lublin.pl

†xavier@ecm.ub.es

${ }^{\ddagger}$ roca@ecm.ub.es

§mariocentelles@ub.edu
}

in nuclear physics and astrophysics, because it governs at the same time important properties of very small entities like atomic nuclei and of very large objects like neutron stars [3]. One of the crucial properties of the symmetry energy, which still is not sufficiently well constrained, is its dependence on the nuclear density. It is relevant in the astrophysical context to understand a wealth of phenomena [3-5], including supernova explosions, neutrino emission, and the cooling mechanism of protoneutron stars, as well as mass-radius relations in neutron stars. Moreover, the density content of the symmetry energy eventually relates to basic issues in physics. This is the case of precision tests of the standard model through atomic parity nonconservation observables [6], and even of studies on constraining a possible time variation of the gravitational constant [7]. In terrestrial laboratories, the available tools to delineate the density dependence of the symmetry energy at saturation and subsaturation densities include the interaction potential between neutron-rich nuclei [8], observables like isospin diffusion and isoscaling in heavy-ion reactions at intermediate energies [9-21], different modes of collective excitations of nuclei [22-26], and, of course, data on the binding and structure of neutron-rich nuclei and on their neutron skin thickness.

In the literature there exist several theoretical formulations to investigate the neutron skin thickness of neutron-rich nuclei and its connections with the symmetry energy. This is the case, for instance, of methods based on the droplet model [27,28], on the concept of surface symmetry energy [5,29,30], thermodynamical arguments [31], nucleonic density form factors [32], mean-field analyses [33-35], or studies in the spirit of the Landau-Migdal approximation [36]. It has been shown that the neutron skin thickness in heavy nuclei, like ${ }^{208} \mathrm{~Pb}$, calculated in mean-field models with either nonrelativistic or relativistic effective nuclear interactions, displays a linear correlation with the slope of the neutron 
equation of state (EOS) obtained with the same interactions at a neutron density $\rho \approx 0.10 \mathrm{fm}^{-3}$ [37-39]. A similar correlation exists between $\Delta R_{n p}$ and the density derivative of the bulk symmetry energy [13-16,33,40,41], as the latter is a measure of the pressure difference between neutrons and protons. These correlations have been exploited in recent years to gain a deeper understanding of the isospin properties of the effective nuclear interaction and to relate them with nuclear and astrophysical observations.

The rms radius of neutron densities in nuclei has been measured with hadronic probes such as proton-nucleus elastic scattering [42-45] or inelastic scattering excitation of the giant dipole and spin-dipole resonances [46,47]. Antiprotonic atoms are helpful to probe the size of the neutron skin of nuclei from the fact that the nuclear periphery is very sensitive to antiprotons in the normally electronic shell. Experimentalists combine two different techniques in this case [48-50], namely the measurement of the antiprotonic $x$ rays that determine the atomic level shifts and widths due to the strong interaction and the radiochemical analysis of the yields after the antiproton annihilation. The values of the neutron skin thickness of 26 stable nuclei from ${ }^{40} \mathrm{Ca}$ to ${ }^{238} \mathrm{U}$ deduced from antiprotonic atoms data by Trzcińska et al. [48,49] follow a roughly linear trend with the overall relative neutron excess $I=$ $(N-Z) / A$ of these nuclei. This trend can be fitted by the relationship $\Delta R_{n p}=(0.90 \pm 0.15) I+(-0.03 \pm 0.02) \mathrm{fm}$ as discussed in Refs. [48,49]. As mentioned, all neutron skin thickness measurements have relatively large uncertainties in comparison with charge radii, and sometimes the results from different experimental techniques are not totally consistent among them $[47,49]$. The neutron skin sizes determined in Refs. [48,49] from the analysis of antiprotonic atoms are to date the largest set of uniformly measured values of $\Delta R_{n p}$ all over the periodic table $(40 \leqslant A \leqslant 238)$. Due to this reason, we shall use hereinafter these data as the experimental benchmark for our calculations.

The droplet model (DM) describes in a physically transparent way nuclear radii and relates them directly with basic properties of the nuclear interactions. In the present article we study the neutron skin thickness of atomic nuclei with the DM using various effective nuclear interactions of the Skyrme, Gogny, and relativistic mean-field (RMF) type. The present work extends with a new analysis and perspective a first presentation of our study made in Ref. [51]. Here, we will show that the ratio of the DM parameters $J$ and $Q$, which drives the value of the neutron skin thickness in heavy nuclei, is correlated with the slopes in density of the nuclear symmetry energy and of the EOS of neutron matter. We compare the DM values for the neutron skin thickness with the results obtained in self-consistent extended Thomas-Fermi (ETF) calculations of finite nuclei [52-55], because both methods are free of shell effects. A non-negligible role of the contribution of the difference in the surface widths of the neutron and proton density profiles is noticed. Next, we use the experimental neutron skin thickness measured in antiprotonic atoms to explore the range of possible values of the ratio $J / Q$ that are favored by neutron skins. With these values we can predict some properties of the density dependence of the nuclear symmetry energy. Our results are compared with the constraints recently obtained in the literature using other observables and methods.

The present article is arranged as follows. In the second section we study the neutron skin thickness of heavy nuclei on the basis of the DM $[27,56,57]$ and show a correlation that links the value of the ratio $J / Q$, which governs the neutron skin thickness of nuclei, with the slope of the symmetry energy in bulk matter at saturation. In the third section, the contribution of the surface widths of the neutron and proton density distributions to the neutron skin thickness is analyzed with the DM using nonrelativistic and covariant mean-field nuclear interactions. In the fourth section we estimate possible constraints on the density dependence of the nuclear symmetry energy on the basis of the DM and the experimental data on neutron skin sizes derived from antiprotonic atoms. We discuss the present results in comparison with the recent constraints obtained from various observables and methods. Finally, the summary and our conclusions are laid in the fifth section. We outline the procedure for the calculation of the $Q$ coefficient in the Appendix.

\section{THE FRAMEWORK}

\section{A. Neutron skin thickness in the droplet model}

In the DM of average nuclear properties [52,56,57] the neutron skin thickness of a finite nucleus is computed from the expression $[27,56]$

$$
\Delta R_{n p}=\sqrt{\frac{3}{5}}\left[t-\frac{e^{2} Z}{70 J}+\frac{5}{2 R}\left(b_{n}^{2}-b_{p}^{2}\right)\right],
$$

where $e^{2} Z / 70 \mathrm{~J}$ is a correction due to the Coulomb interaction, $R=r_{0} A^{1 / 3}$ is the nuclear radius, and $b_{n}$ and $b_{p}$ are the surface widths of the neutron and proton density profiles. In the "standard" version of the DM it is assumed that $b_{n}=b_{p}=1 \mathrm{fm}[27,28,56]$, which implies a vanishing surface width correction to the neutron skin thickness.

The quantity $t$ in Eq. (2) represents the distance between the neutron and proton mean surface locations. This distance is computed as $[27,56]$

$$
t=\frac{3}{2} r_{0} \frac{J}{Q} \frac{I-\frac{c_{1} Z}{12 J} A^{-1 / 3}}{1+\frac{9}{4} \frac{J}{Q} A^{-1 / 3}},
$$

where $I=(N-Z) / A, J$ is the symmetry energy coefficient at saturation, $Q$ is the surface stiffness coefficient, and $c_{1}=3 e^{2} / 5 r_{0}$. The coefficient $J$ represents with a very good accuracy the energy cost per nucleon to convert all protons into neutrons in symmetric infinite nuclear matter at saturation density $\rho_{0}$. The surface stiffness coefficient $Q$ measures the resistance of the system against separation of neutrons from protons to form a neutron skin. To extract $Q$ from an effective nuclear interaction requires performing calculations of asymmetric semi-infinite nuclear matter (ASINM). Therefore, the calculated value of $Q$ may depend somewhat on the type of approach, such as the Hartree-Fock or Thomas-Fermi methods, employed to describe the nuclear surface [30,52,53,58-60].

From Eq. (3), one sees that the leading contribution to $t$ in large nuclei is the term $\frac{3}{2} r_{0}(J / Q) I$. Thus, the DM suggests 
TABLE I. Saturation density $\rho_{0}$ and $J, L, K_{\text {sym }}$, and $Q$ parameters of the Skyrme and Gogny forces as well as RMF parameter sets used in this work.

\begin{tabular}{lcccccc}
\hline \hline Force & $\begin{array}{c}\rho_{0} \\
\mathrm{fm}^{-3}\end{array}$ & $\begin{array}{c}J \\
\mathrm{MeV}\end{array}$ & $\begin{array}{c}L \\
\mathrm{MeV}\end{array}$ & $\begin{array}{c}K_{\text {sym }} \\
\mathrm{MeV}\end{array}$ & $\begin{array}{c}Q \\
\mathrm{MeV}\end{array}$ & $J / Q$ \\
\hline SGII & 0.158 & 26.83 & 37.7 & -146 & 41.7 & 0.64 \\
SVI & 0.144 & 26.88 & -7.3 & -471 & 78.4 & 0.34 \\
SIII & 0.145 & 28.16 & 9.9 & -394 & 63.6 & 0.44 \\
T6 & 0.161 & 29.97 & 30.9 & -211 & 47.8 & 0.63 \\
SkP & 0.163 & 30.00 & 19.7 & -267 & 52.1 & 0.58 \\
SkM* & 0.160 & 30.03 & 45.8 & -156 & 39.0 & 0.77 \\
SkX & 0.155 & 31.10 & 33.2 & -252 & 56.2 & 0.55 \\
NL3 $\Lambda_{v}=0.03$ & 0.148 & 31.68 & 55.3 & -8 & 45.2 & 0.70 \\
D1S & 0.163 & 31.93 & 22.4 & -252 & $53^{\mathrm{a}}$ & 0.60 \\
SLy4 & 0.160 & 32.00 & 46.0 & -120 & 46.1 & 0.69 \\
FSUGold & 0.148 & 32.59 & 60.5 & -52 & 43.7 & 0.75 \\
NL3 $\Lambda_{v}=0.02$ & 0.148 & 33.15 & 68.2 & -54 & 39.6 & 0.84 \\
NL3 $\Lambda_{v}=0.01$ & 0.148 & 34.96 & 87.7 & -46 & 35.2 & 0.99 \\
NL-SH & 0.146 & 36.12 & 113.7 & 80 & 34.5 & 1.05 \\
TM1 & 0.145 & 36.89 & 110.8 & 34 & 34.3 & 1.08 \\
NL3 & 0.148 & 37.40 & 118.5 & 101 & 31.7 & 1.18 \\
NL1 & 0.152 & 43.46 & 140.2 & 143 & 29.4 & 1.48 \\
NL2 & 0.146 & 45.12 & 133.4 & 20 & 41.7 & 1.08 \\
\hline \hline
\end{tabular}

${ }^{\text {a Estimated value. }}$

that one can expect a correlation between $\Delta R_{n p}$ and $J / Q$ in heavy nuclei. We illustrate this fact in the left panel of Fig. 1. We depict there the neutron skin thickness of ${ }^{208} \mathrm{~Pb}$ obtained from self-consistent quantal calculations with the Skyrme and Gogny Hartree-Fock methods as well as with the RMF Hartree approach. The results are shown as a function of the value of the $J / Q$ ratio for various mean-field effective interactions. The $J$ values of the selected interactions (about $27-32 \mathrm{MeV}$ in the nonrelativistic forces and about $32-45 \mathrm{MeV}$ in the covariant forces, see Table I) cover widely the plausible physical range of the bulk symmetry energy. The values of $Q$ used in this work have been extracted from ASINM calculations performed in the extended Thomas-Fermi (ETF) approach as described in the Appendix (see also Ref. [53]). Even if the shell effects, present in the mean-field calculations of $\Delta R_{n p}$, are not built in the DM [27], one observes a considerably linear correlation between the values of $\Delta R_{n p}$ and $J / Q$. It should be pointed out that while all the effective interactions have been accurately calibrated to data on binding energies and charge radii, and describe these properties very successfully, they predict widely different values of $\Delta R_{n p}$, as we see in the present case of ${ }^{208} \mathrm{~Pb}$. This underlines the fact that the isospin sector of the effective interactions is little constrained.

\section{B. Properties of the nuclear symmetry energy}

Let us consider the energy per particle $e(\rho, \delta)$ in asymmetric infinite nuclear matter of total density $\rho=\rho_{n}+\rho_{p}$ and relative neutron excess $\delta=\left(\rho_{n}-\rho_{p}\right) / \rho$, where $\rho_{n}$ and $\rho_{p}$ stand for the neutron and proton densities, respectively. The general expression

$$
e(\rho, \delta)=e(\rho, \delta=0)+c_{\text {sym }}(\rho) \delta^{2}+\mathcal{O}\left(\delta^{4}\right)
$$

defines the symmetry energy coefficient $c_{\text {sym }}(\rho)$ of a nuclear EOS at the density $\rho$. This expression is particularly useful because $c_{\text {sym }}(\rho)$ dominates the corrections to the symmetric limit for all values of $\delta$, especially at the subsaturation densities of relevance for finite nuclei [61]. Actually, $c_{\text {sym }}(\rho)$ provides with excellent accuracy the difference between the binding energies of pure neutron matter $(\delta=1)$ and symmetric matter $(\delta=0)$.

It is customary, and insightful, to characterize the behavior of an EOS around the saturation density $\rho_{0}$ by means of a few bulk parameters calculated at the saturation point, as in the formula [10,13-16,56,61-63]

$$
e(\rho, \delta) \approx a_{v}+\frac{K_{v}}{2} \epsilon^{2}+\left[J-L \epsilon+\frac{K_{\text {sym }}}{2} \epsilon^{2}\right] \delta^{2},
$$

where $\epsilon=\left(\rho_{0}-\rho\right) / 3 \rho_{0}$ expresses the relative density displacement from $\rho_{0}$. Here, the quantities $a_{v}$ and $K_{v}$ denote the energy per particle and the incompressibility modulus of symmetric nuclear matter. One has $c_{\text {sym }}\left(\rho_{0}\right)=J$. The DM coefficients $L$ and $K_{\text {sym }}$ are, respectively, proportional to the slope and the curvature of the symmetry energy coefficient $c_{\text {sym }}(\rho)$ at saturation density:

$$
L=\left.3 \rho_{0} \frac{\partial c_{\mathrm{sym}}(\rho)}{\partial \rho}\right|_{\rho_{0}}, \quad K_{\mathrm{sym}}=\left.9 \rho_{0}^{2} \frac{\partial^{2} c_{\mathrm{sym}}(\rho)}{\partial \rho^{2}}\right|_{\rho_{0}} .
$$

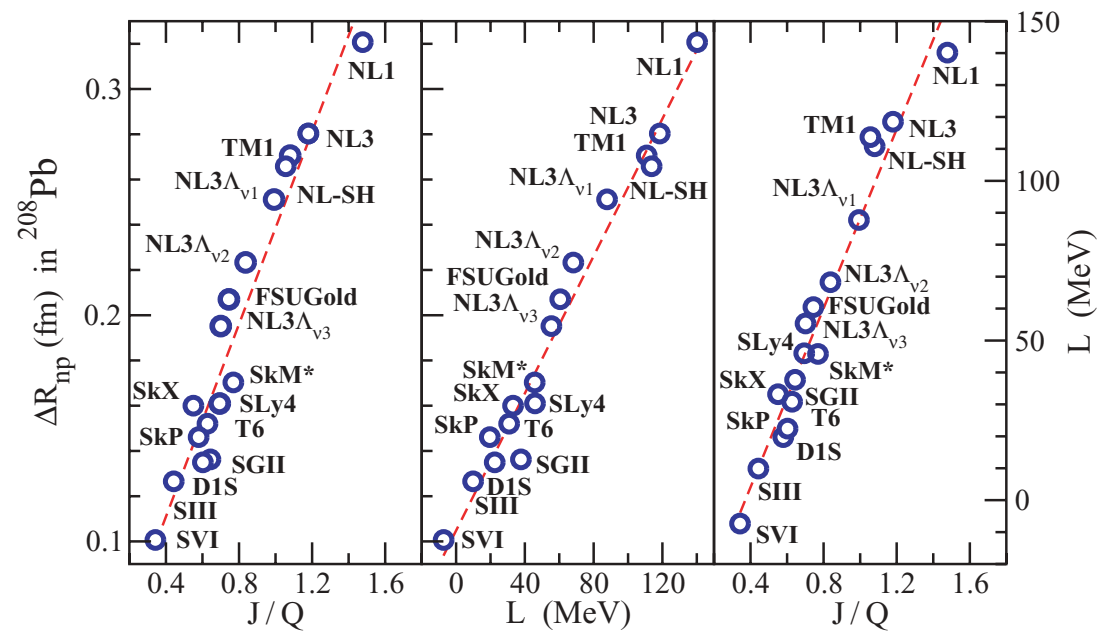

024316-3
FIG. 1. (Color online) For several nuclear mean-field models, existing correlation between the neutron skin thickness $\Delta R_{n p}$ in ${ }^{208} \mathrm{~Pb}$ and the ratio $J / Q$ (left panel) and between $\Delta R_{n p}$ in ${ }^{208} \mathrm{~Pb}$ and the slope of the symmetry energy $L$ (middle panel). The correlation between the coefficient $L$ and the ratio $J / Q$ is also shown (right panel). In the present figure, $\Delta R_{n p}$ has been computed with Eq. (1) from the rms radii of quantal self-consistent calculations for the indicated mean-field models. 
The quadratic expansion $c_{\text {sym }}(\rho) \approx J-L \epsilon+\frac{1}{2} K_{\text {sym }} \epsilon^{2}$ in Eq. (5) is often a reliable representation of the actual value of the $c_{\text {sym }}(\rho)$ coefficient at densities roughly between $\rho_{0} / 2$ and $2 \rho_{0}$ [61]. For instance, in the case of a typical subsaturation density value $\rho=0.10 \mathrm{fm}^{-3}$, one finds that the above quadratic expansion of $c_{\text {sym }}(\rho)$ differs from the exact $c_{\text {sym }}(\rho)$ by less than $1 \%$ in many different nuclear mean field forces [51]. These facts point to the usefulness of investigating parameters such as $L$ and $K_{\text {sym }}$ for the characterization of the density dependence of the symmetry energy.

The values of the DM coefficients $J, L$, and $K_{\text {sym }}$ for the nonrelativistic forces and the RMF parameter sets considered in this work are given in Table I. In the Skyrme and Gogny effective interactions the symmetry energy coefficient at saturation $J$ takes values around $30 \mathrm{MeV}$. The RMF parametrizations have larger values of $J$, also with a larger spread. The slope $(L)$ and the curvature $\left(K_{\text {sym }}\right)$ of the symmetry energy at saturation take even more widely scattered values among the different interactions. The consequence is that all mentioned nuclear models predict a different behavior of the symmetry energy at subsaturation densities, what can be seen, e.g., in Fig. 1 of Ref. [13].

As is known, the density dependence of the symmetry energy near saturation tends to be much softer in the nonrelativistic forces than in the covariant meson-exchange models of nuclear structure (see the values of $L$ in Table I). In particular, taking into account Eq. (5), the slope of the neutron EOS

$$
\frac{d e(\rho, \delta=1)}{d \rho}=\frac{L}{3 \rho_{0}}-\frac{K_{v}+K_{\mathrm{sym}}}{3 \rho_{0}} \epsilon,
$$

and of the symmetry energy

$$
\frac{d c_{\mathrm{sym}}(\rho)}{d \rho}=\frac{L}{3 \rho_{0}}-\frac{K_{\mathrm{sym}}}{3 \rho_{0}} \epsilon,
$$

calculated at densities $\rho$ close to the saturation value $\rho_{0}$ differ considerably between models. We also know that $\Delta R_{n p}$ in ${ }^{208} \mathrm{~Pb}$ shows a linear dependence with these slopes at some subsaturation density $\rho \simeq 0.10 \mathrm{fm}^{-3}$ [37-40]. Therefore it is reasonable that the neutron skin thickness in ${ }^{208} \mathrm{~Pb}$ and the leading term $L / 3 \rho_{0}$ of Eqs. (7) and (8) are related. As far as $\rho_{0}$ does not change much in the different effective forces, a correlation between $\Delta R_{n p}$ in ${ }^{208} \mathrm{~Pb}$ and the DM coefficient $L$ is thus expected $[13-15,33,41]$ and we display it in the middle panel of Fig. 1.

From the discussed results of $\Delta R_{n p}$ versus $J / Q$ and of $\Delta R_{n p}$ versus $L$ in Fig. 1, a correlation between the DM coefficient $L$ and the $J / Q$ ratio is to be expected too. Note that $L$ rules the density dependence of the symmetry energy of the nuclear equation of state [Eq. (8)] and that $Q$ governs the thickness of the neutron skin of finite nuclei [Eqs. (2) and (3)]. The correlation between $L$ and $J / Q$ can be seen in the right panel of Fig. 1. This correlation shows that the value of the slope $L$ of the symmetry energy at the saturation density increases with the value of the ratio between the bulk symmetry energy coefficient $J$ and the surface stiffness coefficient $Q$. The trend is considerably linear among the various nuclear effective interactions.

Previous literature [53,59] has shown that the systematics of experimental binding energies relates increasing values of
$J$ with decreasing values of $Q$ in nuclear effective interactions whose parameters have been adjusted to describe experimental data. We note this same trend in Table I, where the RMF sets that in general have larger $J$ values also tend to have smaller $Q$ values than their nonrelativistic counterparts. A smaller $Q$ coefficient means that it is easier to develop a neutron skin in finite nuclei. Consistently, the neutron skin thickness in ${ }^{208} \mathrm{~Pb}$ (or any other heavy nucleus) is usually larger when computed with a RMF parameter set than when computed with a nonrelativistic force. These facts, and the noticed correlation between $L$ and $J / Q$, allow one to interpret in a qualitative way within the DM, the correlation pointed out in Refs. [37-40] between the slope of the symmetry energy at some subsaturation density $\rho \simeq 0.10 \mathrm{fm}^{-3}$ and the neutron skin thickness in a heavy nucleus. In a recent work [51] we have investigated further the relations of the neutron skin thickness with the parameters $L$ and $K_{\text {sym }}$ that characterize the density dependence of the symmetry energy around saturation.

\section{SURFACE WIDTH CONTRIBUTION TO THE NEUTRON SKIN THICKNESS}

The neutron skin thickness values derived from measurements performed in antiprotonic atoms have been obtained in Refs. [48,49]. It is assumed that the neutron skin is due to an enhancement of the neutron surface width with respect to the proton surface width and that the mean location of the proton and neutron surfaces in these nuclei are the same. This situation corresponds to the so-called "neutron halo-type" distribution [48]. It has been shown that the same set of experimental values of neutron skin thickness can be explained with similar quality as in Ref. [48] by means of the "standard" version of the DM (where $b_{n}=b_{p}$ ) [28]. The latter case assumes that the peripheral neutrons are concentrated at the neutron surface, which is shifted with respect to the proton surface and that both the neutron and proton density distributions have the same surface width. This is rather the pattern of the so-called "neutron skin-type" distribution according to Ref. [48].

The analysis of neutron and proton densities calculated with nuclear mean-field interactions carried out in Ref. [32] by means of the Helm model points out that self-consistent mean-field densities show a mixed character between the "neutron halo" and "neutron skin" patterns. This means that, actually, the self-consistent neutron and proton density profiles obtained with nuclear effective interactions differ not only in the mean location of their surfaces but also in their surface widths. In the following we shall see that similar conclusions are found from the calculations of the neutron skin thickness performed in the DM with formula (2). It will turn out that the surface width contribution $\propto\left(b_{n}^{2}-b_{p}^{2}\right)$ in the DM expression (2) for the neutron skin thickness, which arises from $b_{n} \neq b_{p}$, is necessary to reproduce the neutron skin thickness values calculated from the definition (1) using self-consistent densities of finite nuclei obtained with the ETF approach in mean-field theory, for nonrelativistic forces as well as for relativistic parametrizations.

In Fig. 2 we display by empty symbols, as a function of the overall relative neutron excess $I=(N-Z) / A$, the neutron 


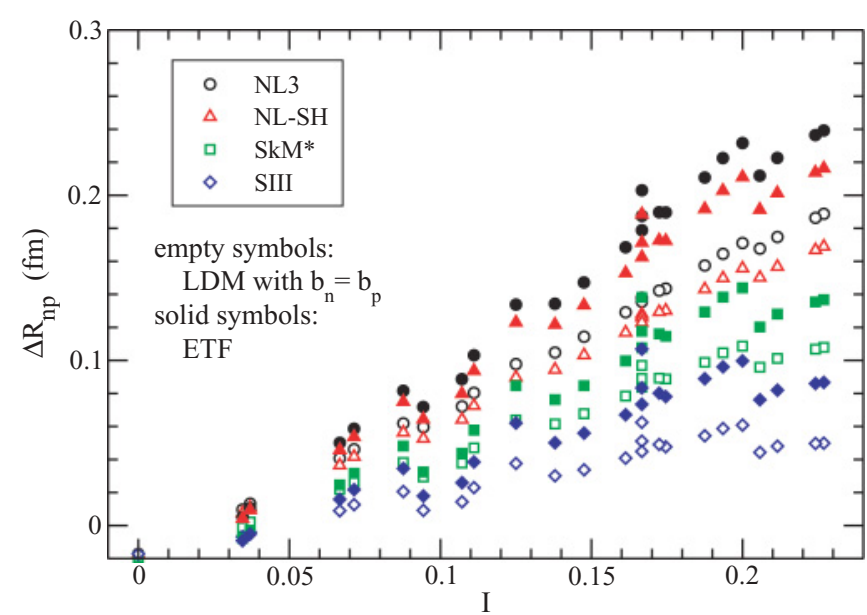

FIG. 2. (Color online) The neutron skin thickness predicted by the "standard" version of the DM [Eq. (2) with $b_{n}=b_{p}$ ] is compared with the result obtained from self-consistent ETF calculations of finite nuclei, in four illustrative mean-field parameter sets. The nuclei considered are those investigated experimentally with antiprotonic atoms in Refs. [48,49] and have masses $40 \leqslant A \leqslant 238$.

skin thickness predicted by the "standard" version of the DM [namely Eq. (2) with $b_{n}=b_{p}$ ] using some well-known effective forces. The nuclei are those from ${ }^{40} \mathrm{Ca}$ to ${ }^{238} \mathrm{U}$ measured in the experiments with antiprotonic atoms [48,49], and that were studied with the DM in Ref. [28]. The values shown in Fig. 2 have been computed using the SIII and SkM* Skyrme forces and the NL-SH and NL3 RMF parameter sets, as suitable examples. We have chosen these four parameter sets for display in Fig. 2 because they span the whole range of values of the ratio $J / Q$ of nuclear interactions that describe reasonably well the ground-state properties of finite nuclei, having a bulk symmetry energy coefficient $J$ between 28 and $37 \mathrm{MeV}$ (see Fig. 1 and Table I). The DM results for $\Delta R_{n p}$ obtained with the other mean-field interactions considered in this work that have a $J$ coefficient between the values of SIII and NL3, also lie within the window of results delimited by the SIII and NL3 interactions in Fig. 2.

The values of $\Delta R_{n p}$ predicted by the DM are compared in Fig. 2 with the values that we obtain from self-consistent ETF calculations in finite nuclei (filled symbols). Both models do not incorporate shell effects. In Fig. 2 we have used the ETF approach in the non-relativistic [54] and relativistic [55] frameworks to compute $\Delta R_{n p}$. We have calculated these ETF values of $\Delta R_{n p}$ by application of Eq. (1) with the rms radii of the self-consistent neutron and proton densities obtained in each isotope. From Fig. 2 two significant points stem. First, the predictions of the DM in the "standard" form $\left(b_{n}=b_{p}\right)$ systematically undershoot the ETF neutron skin thickness computed in finite nuclei with the selected effective nuclear interactions. In particular, this trend is reinforced with growing neutron excess $I$. Second, it can be observed that for a given nucleus the difference between the ETF value of $\Delta R_{n p}$ computed with (1) and the value provided by the "standard" DM prescription is slightly larger in the RMF parameter sets than in the Skyrme forces. Altogether, these facts suggest that in the mean-field interactions the surface width contribution

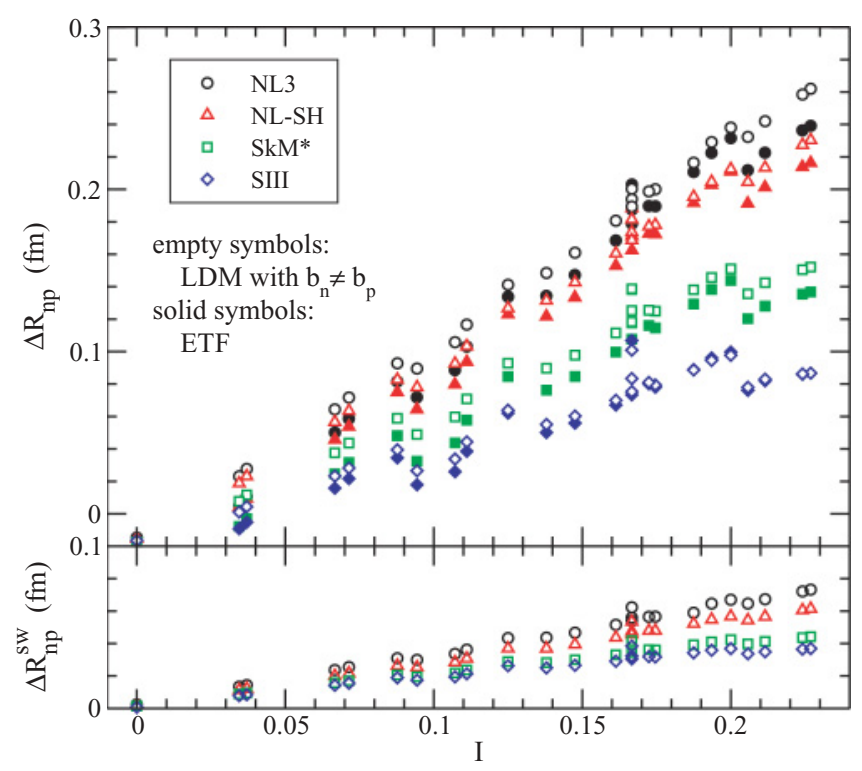

FIG. 3. (Color online) (Upper panel) The same as in Fig. (2) but here the DM values include a nonvanishing surface width contribution $\Delta R_{n p}^{\mathrm{sw}}$ [Eq. (10)] with $b_{n}$ and $b_{p}$ obtained from ASINM calculations as described in the text. (Lower panel) The surface width contribution $\Delta R_{n p}^{\mathrm{sw}}$ (the vertical scale proportionality is the same as in the upper panel).

to the DM formula for $\Delta R_{n p}$ does not vanish and that this contribution has some dependence on the ratio $J / Q$ of the force.

To apply the full Eq. (2) to compute the neutron skin thickness including the surface width correction, one needs to evaluate the neutron and proton surface widths in finite nuclei. In practice, there is not conclusive experimental evidence on the difference of the surface widths $b_{n}$ and $b_{p}$ of the nuclear density distributions $[27,28,32,50,52]$. To estimate $b_{n}^{2}-b_{p}^{2}$ we will therefore rely on theoretical guidance as a surrogate. Within the context related to the DM and the leptodermous expansion of a finite nucleus [52,56,57], the surface properties in finite nuclei can be extracted from ASINM calculations. The semi-infinite geometry does not include shell, Coulomb, or finite-size effects. We will use here the ETF method including $\hbar^{2}$ corrections for describing ASINM, because the ETF method is free of the Friedel oscillations of the quantal densities $[30,60]$. We summarize in the Appendix the basic aspects of the procedure. More details can be found in Ref. [53]. From Eqs. (A1)-(A4) of the Appendix, we can obtain the values of the surface widths $b_{n}$ and $b_{p}$ in ASINM with a given relative neutron excess in the bulk $\delta_{0}$. In the DM, these surface widths correspond to the values $b_{n}$ and $b_{p}$ in finite nuclei if $\delta_{0}$ is calculated from the overall relative neutron excess $I$ of the nucleus through the following relation $[52,53,56]$ :

$$
\delta_{0}=\frac{I+\frac{3}{8} \frac{c_{1}}{Q} \frac{Z^{2}}{A^{5 / 3}}}{1+\frac{9}{4} \frac{J}{Q} A^{-1 / 3}},
$$

which takes into account the Coulomb correction.

Once the neutron and proton surface widths in finite nuclei are known, we can compute their contribution to the neutron 
skin thickness, which reads [cf. Eq. (2)]

$$
\Delta R_{n p}^{\mathrm{sw}}=\sqrt{\frac{3}{5}} \frac{5}{2 R}\left(b_{n}^{2}-b_{p}^{2}\right) .
$$

The corresponding values of $\Delta R_{n p}^{\mathrm{sw}}$ for the nuclei considered in Fig. 2 are displayed in the bottom panel of Fig. 3. It is worth pointing out that the calculated $\Delta R_{n p}^{\mathrm{sw}}$ values show, for each nuclear interaction, a well-defined increasing linear trend as a function of the overall relative neutron excess $I$ of the nuclei.

The neutron skin thickness predictions of the DM when one includes the surface width contribution (2) are displayed in the top panel of Fig. 3 by empty symbols. Note that the results correspond to adding the values $\Delta R_{n p}^{\mathrm{sw}}$ shown in the bottom panel of this figure to the DM values that we have displayed in Fig. 2. As done in Fig. 2, we compare in the top panel of Fig. 3 the DM results with the self-consistent ETF calculations of $\Delta R_{n p}$ [54,55]. One now observes an improved and remarkable agreement between the DM predictions and the self-consistent ETF values computed with the same interaction, stemming from the inclusion of the calculated $\Delta R_{n p}^{\mathrm{sw}}$ contribution. It is interesting to note that the neutron skin thickness obtained with Eq. (1) from the rms radii of the self-consistent ETF calculations in finite nuclei shows a well defined increasing linear tendency with the relative neutron excess $I$, similarly to the case of the results of the DM and in consonance with the trend of the experimental values derived from antiprotonic atoms [48].

The lower panel of Fig. 3 also suggests that, for a given nucleus, $\Delta R_{n p}^{\mathrm{sw}}$ grows when the $J / Q$ ratio of the nuclear interaction increases (see Table I). To analyze this behavior in more detail, we fit $\Delta R_{n p}^{\mathrm{sw}}$ by means of a law $\sigma^{\mathrm{sw}} I$, which defines the slope $\sigma^{\mathrm{sw}}$ of $\Delta R_{n p}^{\mathrm{sw}}$ with respect to the relative neutron excess $I$. This slope is displayed in Fig. 4 as a function of the $J / Q$ ratio for different interactions. The slopes $\sigma^{\text {sw }}$ lie inside a band limited by two straight lines, corresponding to the equations $\sigma^{\mathrm{sw}}=0.3 \mathrm{~J} / Q+0.07 \mathrm{fm}$ (left) and $\sigma^{\mathrm{sw}}=$ $0.3 \mathrm{~J} / Q-0.05 \mathrm{fm}$ (right). Note that all considered Skyrme forces have slopes $\sigma^{\mathrm{sw}}$ below $0.25 \mathrm{fm}$, whereas the analyzed RMF models have slopes $\sigma^{\text {sw }}$ always above this value.

One relevant conclusion is that bulk and finite nuclei properties described through successful theoretical mean-field models constrain the possible values of the surface width contribution to the neutron skin thickness between the limits portrayed in Fig. 4. From this figure it can be deduced that the surface width contribution to the neutron skin thickness $\Delta R_{n p}^{\mathrm{sw}}$ has, on top of a global increasing trend with $J / Q$, a more involved dependence on the parameters of the effective nuclear interactions. For instance, on the one hand, the RMF force FSUGold and the Skyrme force SkM* have almost the same $J / Q$ ratio (see Table I). However, the predicted values of $\sigma^{\text {sw }}$ are clearly different for both interactions as can be seen in Fig. 4. On the other hand, it is possible to find different interactions that have almost the same slope $\sigma^{\mathrm{sw}}$, and therefore the same $\Delta R_{n p}^{\mathrm{sw}}$, but with different values of the $J / Q$ ratio. Some examples of this fact in Fig. 4 are the Skyrme forces SIII and SGII (with slopes of 0.16 and $0.15 \mathrm{fm}$, respectively) and the RMF parametrizations FSUGold and NL3 (with slopes $0.30 \mathrm{fm}$ and $0.31 \mathrm{fm}$ ). These facts suggest that it is possible to find the same total neutron skin thickness by combining a

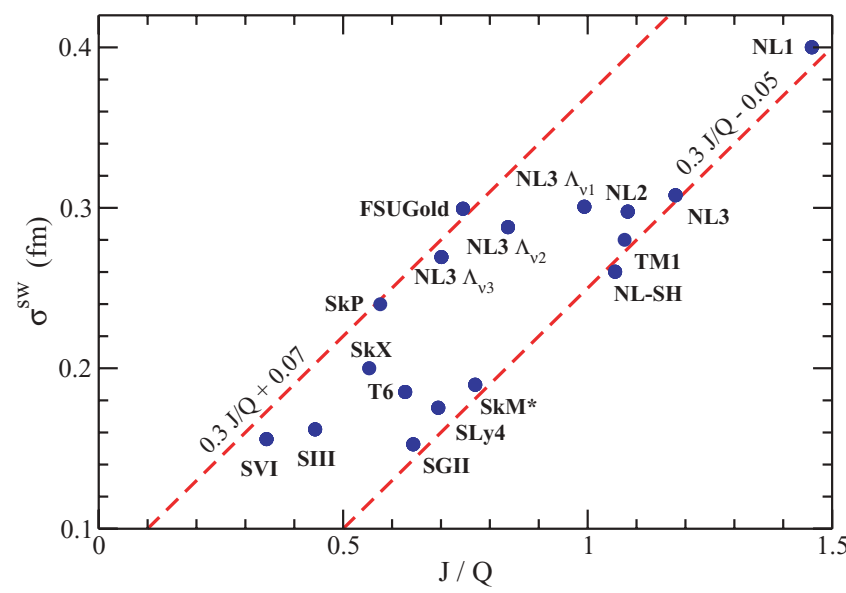

FIG. 4. (Color online) Average slope of $\Delta R_{n p}^{\mathrm{sw}}$ with respect to $I$ for various nuclear mean-field models as a function of $J / Q$. All the data lie in the area limited by the marked lines $\sigma^{\mathrm{sw}}=0.3 \mathrm{~J} / Q+0.07 \mathrm{fm}$ and $\sigma^{\mathrm{sw}}=0.3 \mathrm{~J} / Q-0.05$.

small value of the $J / Q$ ratio in $t$ [see Eq. (3)] with a large $\Delta R_{n p}^{\mathrm{sw}}$ contribution or, vice versa, by combining a large $J / Q$ ratio in $t$ with a small $\Delta R_{n p}^{\mathrm{sw}}$ contribution.

\section{ESTIMATES OF THE DENSITY CONTENT OF THE SYMMETRY ENERGY}

As we have pointed out, the nuclear symmetry energy and the properties of the EOS of neutron-rich matter are of increasing importance in both nuclear physics and astrophysics. There is a significant recent effort in the community toward constraining the values of the parameters that characterize the density dependence of the symmetry energy in the subsaturation regime of the EOS. The new developments come in particular from the investigation of isospin-sensitive observables in intermediate-energy heavy-ion collisions [9-21] and in nuclear resonances [22-25]. Obviously, the different studies do not deal with exactly the same regimes of density and energy. One also has to keep in mind that the connection of the experiments with the EOS is not at all trivial; it often requires extrapolations of the measured data, which imply a model dependence. Therefore, it is important to further investigate indications from those and other experimental probes of the symmetry energy, with different methodologies, as well as to study the interplay between the constraints derived from the different analyses.

In the present section we want to apply the experience gained in the DM study of the neutron skin thickness performed in the previous sections to estimate possible constraints on the density dependence of the nuclear symmetry energy as suggested by neutron skin data. To this end, we shall first obtain the range of values of the $J / Q$ ratio that are compatible with the neutron skin thickness derived from the experimental data in antiprotonic atoms. We recall that this set of data for 26 stable nuclei is to date the largest set of uniformly measured neutron skins spanning the mass table. Once the estimated range of values for the ratio $J / Q$ will be found, the constraints derived from the neutron skin data on the DM parameter $L$, 
related to the density derivative of the symmetry energy, will be determined from the existing linear correlation between the values of $L$ and $J / Q$. This correlation has been displayed in Fig. 1 and is a general feature of mean-field interactions that have been adjusted to reproduce with good accuracy binding energies and charge radii (often among other properties) of nuclei across the periodic table.

\section{A. Constraints on the $J / Q$ ratio}

From the previous section we know that the surface width part in Eq. (2) gives a non-negligible contribution to neutron skins in effective nuclear interactions. This contribution is needed to reproduce the neutron skin thickness values computed self-consistently in ETF calculations of finite nuclei. We have also seen that to leading order, both the mean location of the neutron and proton surfaces (3) and the surface width correction (10) are basically driven by the value of the $J / Q$ ratio. The discussions in the previous sections suggest to fit the experimental $\Delta R_{n p}^{\exp }$ data by means of the following DM inspired ansatz:

$$
\Delta R_{n p}=\sqrt{\frac{3}{5}}\left(t-\frac{e^{2} Z}{70 J}\right)+\left(0.3 \frac{J}{Q}+c\right) I,
$$

where $t$ is given by Eq. (3). The second term is the surface width contribution. It is parameterized to reproduce the dashed lines on Fig. 4, with $c=-0.07 \mathrm{fm}$ or $c=-0.05 \mathrm{fm}$.

With the ansatz (11), we will use $J / Q$ as an open parameter. It will be constrained by a least-squares minimization from the experimental values $\Delta R_{n p}^{\exp }$ derived from the analysis of antiprotonic atoms [48,49]. We note that Eq. (11), as well as $t$ given by Eq. (3), depends on the particular values of the symmetry energy at saturation $J$ and of the nuclear matter radius $r_{0}$. We fix these quantities to the empirical values $J=31.6 \mathrm{MeV}$ and $r_{0}=1.143 \mathrm{fm}$ (the latter corresponds to a saturation density $\left.\rho_{0}=0.16 \mathrm{fm}^{-3}\right)$. We consider the values $c=0.07 \mathrm{fm}$ and $c=-0.05 \mathrm{fm}$ in Eq. (11), discussed in connection with Fig. 4, to simulate the upper and lower bounds of the window of the theoretical predictions for $\sigma^{\text {sw }}$ obtained according to mean-field models of nuclear structure. In the $\chi^{2}$ minimization we have weighted each $\Delta R_{n p}^{\exp }-\Delta R_{n p}$ difference by the inverse of the associated experimental uncertainties. That is, in practice we have minimized the quantity

$$
\sum_{i}\left[\frac{\Delta R_{n p}(i)-\Delta R_{n p}^{\exp }(i)}{\xi_{i}}\right]^{2}
$$

where $\Delta R_{n p}$ is calculated with Eq. (11) and the $\xi_{i}$ denote the uncertainties of the experimental data.

The fits to experiment give $J / Q=0.667 \pm 0.047$ with $c=0.07 \mathrm{fm}$ and $J / Q=0.791 \pm 0.049$ with $c=-0.05 \mathrm{fm}$ (i.e., a range $0.62 \lesssim J / Q \lesssim 0.84$ ). The quoted uncertainties in the $J / Q$ predictions correspond to the value of one standard deviation associated with the fit made through Eq. (12). To check our method of minimization and error estimation, we have applied the same procedure to make a linear fit $m I+n$ of the experimental data $\Delta R_{n p}^{\exp }$. In this case we have obtained $\Delta R_{n p}=(0.901 \pm 0.147) I+(-0.034 \pm 0.023) \mathrm{fm}$, which fully agrees with the result quoted by the experimentalists $[48,49]$.

The results for $\Delta R_{n p}$ from our fits compared with the experimental data $\Delta R_{n p}^{\exp }$ are displayed as a function of $I$ in Fig. 5. Both extractions of $J / Q$, for $c=0.07$ and $c=-0.05 \mathrm{fm}$, predict basically the same total neutron skin thickness with a similar quality and they are close to the average $\Delta R_{n p}=(0.90 \pm 0.15) I+(-0.03 \pm 0.02) \mathrm{fm}[48,49]$ of the experimental data. However, the splitting of the neutron skin thickness into a part coming from the distance $t$ and another part coming from the surface width $\Delta R_{n p}^{\mathrm{sw}}$ is different in both cases, as we have discussed in the previous section. Therefore, it becomes clear that the experimental neutron skin thickness data, by themselves, may be able to constrain the total value but not its partition into a bulk and a surface width contribution.

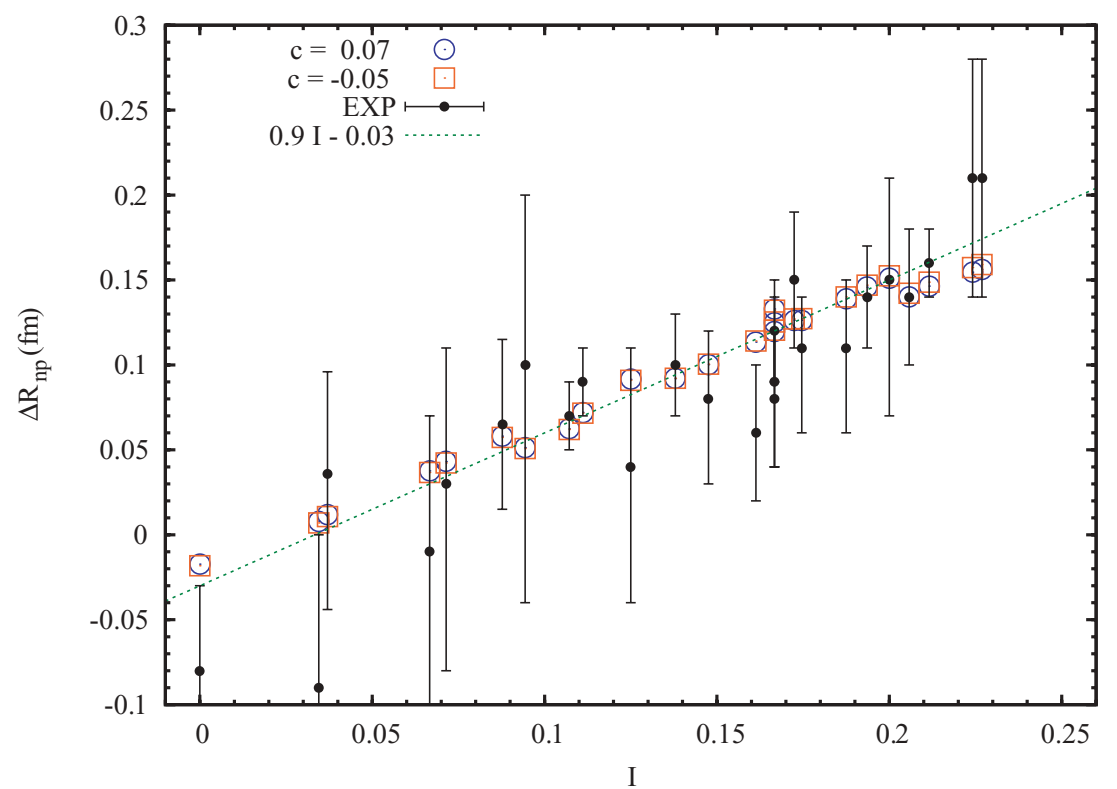

$024316-7$
FIG. 5. (Color online) The values of $\Delta R_{n p}$ obtained from Eq. (11) by fitting the $J / Q$ ratio, using $c=0.07 \mathrm{fm}$ (circles) and $c=-0.05 \mathrm{fm}$ (squares) to reproduce the $\Delta R_{n p}^{\exp }$ values measured in antiprotonic atoms (dots with error bars). The average value of $\Delta R_{n p}^{\exp }$ is marked by the dotted line. 
It is generally acknowledged that the value of the bulk nuclear symmetry energy coefficient $J$ is about $30-32 \mathrm{MeV}$, but there is some uncertainty. To assess the dependence of the extraction of $J / Q$ on the assumed value for the $J$ coefficient (which in the above we have taken as $31.6 \mathrm{MeV}$ ), we have repeated the fit of Eq. (11) to the neutron skin data for $J=35 \mathrm{MeV}$ and for $J=28 \mathrm{MeV}$. The results are, respectively, $J / Q=0.642 \pm 0.046$ and $J / Q=0.701 \pm 0.048$ if $c=0.07 \mathrm{fm}$ and $J / Q=0.764 \pm 0.048$ and $J / Q=0.829 \pm$ 0.051 if $c=-0.05 \mathrm{fm}$. All in all, it seems safe to consider that within the present model the $J / Q$ values compatible with the data from antiprotonic atoms span a window from about $J / Q=0.59$ to $J / Q=0.88$ or $0.6 \lesssim J / Q \lesssim 0.9$ in round figures.

We recall that the DM of nuclei does not incorporate shell effects and averages the corresponding quantal magnitudes. With the method described we have fitted $\Delta R_{n p}^{\exp }$ that in general contains shell effects and possible correlation and deformation contributions. But, as mentioned, the neutron skin data analyzed show a well-defined linear trend with the relative neutron excess $I$ (namely $\Delta R_{n p}=(0.90 \pm 0.15) I+$ $(-0.03 \pm 0.02) \mathrm{fm}[48,49])$. This trend, and the agreement of the DM values for $\Delta R_{n p}$ with the self-consistent ETF calculations in finite nuclei, which also are free of shell effects and have a linear trend with $I$ (Figs. 2 and 3), gives more reliability to the predictions obtained with the DM formula from the experimental data.

\section{B. Constraints on the $L$ parameter}

As we have discussed previously, the parameter $L$ has a direct relation with the slope of the symmetry energy of the nuclear EOS, see Eq. (8). Having determined the values of the $J / Q$ ratio compatible with the experimental neutron skins measured in antiprotonic atoms, we can use the linear correlation between $L$ and $J / Q$ found in mean-field effective nuclear interactions to obtain an insight on the values of the parameter $L$ favored by neutron skins.

We have displayed the linear correlation $L=m J / Q+n$ in the rightmost panel of Fig. 1 (the linear correlation coefficient for the shown interactions is $r=0.978$ ). The values of the $m$ and $n$ coefficients have some dependence on the set of interactions chosen to make the linear regression. We have checked that this dependence is rather weak. Namely we have tested the correlation of $L$ with $J / Q$ by taking into account successively $10,14,18$, and 24 interactions and we have found the linear regressions to be comprised between $L=139 \mathrm{~J} / Q-52 \mathrm{MeV}$ and $L=150 \mathrm{~J} / Q-57 \mathrm{MeV}$. Considering these two limiting cases and the constraint $0.6 \lesssim J / Q \lesssim 0.9$ found in the previous section, leads to a variation of $L$ between 31 and $78 \mathrm{MeV}$. Thus, our estimate for the $L$ coefficient, which takes into account the surface width correction in $\Delta R_{n p}$ obtained in the calculations with mean-field interactions, basically lies in the range $30 \lesssim L \lesssim 80 \mathrm{MeV}$. Had we kept the value of $J$ fixed at $31.6 \mathrm{MeV}$, the extracted range for $L$ would be a little narrower: $35 \lesssim L \lesssim 70 \mathrm{MeV}$.

In a previous work [51] we have investigated the correlations between the symmetry energy coefficient in finite nuclei and in the EOS at subsaturation densities. These correlations allow one to derive an aproximate formula for the neutron skin thickness with explicit dependence on the $L$ coefficient. By comparison of that result for the neutron skin thickness with the experimental data set of Refs. [48,49], in Ref. [51] we found a range of values $L=55 \pm 25 \mathrm{MeV}$ (displayed in Fig. 3 of Ref. [51]) when one includes the surface width contribution $\Delta R_{n p}^{\mathrm{sw}}$ in the calculations. That prediction is consistent with the values obtained here by the present procedure. A somewhat higher range $L=75 \pm 25 \mathrm{MeV}$ was obtained [51] when one neglects $\Delta R_{n p}^{\mathrm{sw}}$. Although a vanishing $\Delta R_{n p}^{\mathrm{sw}}$ value, corresponding to $b_{n}=b_{p}$ in the nucleon density distributions, is not favored by the mean-field interactions (see Sec. III), it cannot be discarded without having more experimental evidence on the value of $b_{n}$ as we have noted in Sec. III (see also Refs. [28,48]).

In recent years, considerable advances in probing experimentally the density dependence of the symmetry energy at subsaturation have been achieved in heavy-ion collisions (HIC) at intermediate energies. It has been found that the symmetry energy can be modelized around the saturation density with reasonable good approximation by [11-20]

$$
c_{\mathrm{sym}}(\rho)=J\left(\frac{\rho}{\rho_{0}}\right)^{\gamma} .
$$

From Eq. (13), one can estimate the parameter $L$ defined in Eq. (6) from the stiffness $\gamma$ of the symmetry energy, as $L=3 \gamma J$. The values for $\gamma$ extracted in the literature from different HIC observables fall in the range $\gamma \sim 0.55-1.05$, which implies $L$ values roughly between 50 and $100 \mathrm{MeV}$. In these studies, the value of $J$ in Eq. (13) normally has been taken equal to 31.6 or $32 \mathrm{MeV}$. The extraction of the equation of state of cold nuclear matter from HIC data is a very complicated task and requires model assumptions [9-21,64-66]; the indicated estimates for $\gamma$ and $L$ may be somewhat modified as more measurements and analyses be performed. The range $30 \lesssim$ $L \lesssim 80 \mathrm{MeV}$ of $L$ values determined here from neutron skins, assuming the dependence of Eq. (13), favors a constraint $0.32 \lesssim \gamma \lesssim 0.84$ for the $\gamma$ exponent. Thus, the result points toward a soft symmetry energy.

Our estimates for the stiffness $\gamma$ can be compared with alternative predictions derived in the recent literature. For instance, our range $0.32 \lesssim \gamma \lesssim 0.84$ overlaps with the values $\gamma \sim 0.55-0.77$ that Danielewicz [29] obtains from the study of binding energies, neutron skins, and isospin analog states of selected nuclei. It also contains the value $\gamma \sim 0.55$ that is inferred from the analysis of neutron-proton emission ratios in HIC carried out by Famiano et al. [20], as well as with the value $\gamma \sim 0.69$ obtained by Shetty et al. [17-19] from isotopic scaling in intermediate-energy nuclear reactions.

The stiffness of the symmetry energy at subsaturation densities also has been investigated from isospin diffusion data in HIC, by means of simulations with an isospinand momentum-dependent transport model with in-medium nucleon-nucleon cross sections [12-16]. In this case the prediction is $0.69 \lesssim \gamma \lesssim 1.05$. It corresponds to a behavior of $c_{\text {sym }}(\rho)$ that nearly ranges between the familiar $\rho^{2 / 3}$ dependence of the purely kinetic symmetry energy of a free Fermi gas, in the lower limit, and linearity in the density 
$\rho$, in the upper limit. The constraints on the stiffness of the symmetry energy derived from isospin diffusion, combined with an analysis of the properties of Skyrme interactions, are found to lead to a constraint $63 \lesssim L \lesssim 113 \mathrm{MeV}$ [12-16]. The predictions from isospin diffusion are thus a little stiffer, though the lower limits of $\gamma$ and $L$ obtained using this method are in agreement with the upper limits of $\gamma$ and $L$ obtained from our study of neutron skins with inclusion of the surface width contribution.

Another valuable reference comes from the celebrated Thomas-Fermi model of Myers and Świątecki $[67,68]$. This model was fitted very precisely to the binding energies of a comprehensive set of 1654 nuclei. It predicts an EOS that leads to a coefficient $L=49.9 \mathrm{MeV}$. Note that if we compare $c_{\text {sym }}(\rho)$ calculated from the EOS of the Thomas-Fermi model with Eq. (13), an exponent $\gamma=0.51$ is obtained. Additional information on the density content of the symmetry energy arises from the constraints on the symmetry pressure $P_{\text {sym }}=$ $\rho_{0} L / 3$ extracted by Klimkiewicz et al. [24] from the properties of pygmy dipole resonances in nuclei. These are indicative of a value $\gamma \sim 0.35-0.65$ if one assumes $P_{\text {sym }}=\rho_{0} \gamma J$ following from Eq. (13) given above. Trippa et al. [25] have obtained the constraint $23.3<c_{\text {sym }}\left(\rho=0.1 \mathrm{fm}^{-3}\right)<24.9 \mathrm{MeV}$ from consideration of the giant dipole resonance in ${ }^{208} \mathrm{~Pb}$, which implies a range $\sim 0.5-0.65$ for the $\gamma$ exponent. We depict in Fig. 6 the estimated ranges of values for the $L$ parameter from the discussed analyses.

In summary, in spite of the discrepancies in the details, the various findings from experimental isospin-sensitive signals, including ours, all agree on a rather soft nuclear symmetry energy at subsaturation densities. Recent studies of pure neutron matter at low densities based on universal properties of dilute Fermi gases lead to a similar conclusion $[69,70]$. One may mention that there exists recent circumstantial evidence [71], derived from $\pi^{-} / \pi^{+}$ratios in central HIC collisions at

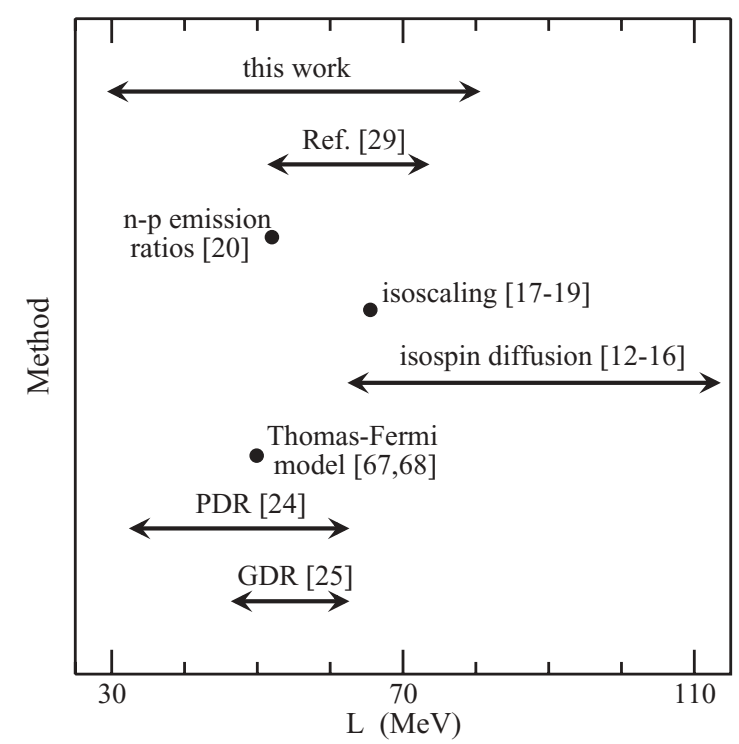

FIG. 6. Comparison of the estimated values of the parameter $L$ from different observables and methods. Some of the estimates have been analyzed through Eq. (13) for $c_{\text {sym }}(\rho)$.
SIS/GSI energies, hinting at that the nuclear symmetry energy is soft also in the region of suprasaturation densities $\left(\rho \geqslant 2 \rho_{0}\right)$. However, further experimental and theoretical confirmations of this fact need to be done [71].

\section{SUMMARY AND CONCLUSIONS}

The droplet model predicts that the neutron skin thickness of atomic nuclei is correlated with the ratio $J / Q$, where $J$ is the symmetry energy in bulk matter and $Q$ is the surface stiffness coefficient. We have shown that the $J / Q$ ratio displays a linear relationship with the DM parameter $L$ in nuclear mean-field models that are calibrated to experimental ground-state properties such as binding energies, charge radii, and single-particle data. In this way, the known correlation between the neutron skin thickness in a heavy nucleus and the density derivative of the symmetry energy (or of the neutron equation of state) evaluated at a subsaturation density, can be interpreted in the context of the DM.

According to the droplet model, the neutron skin thickness is correlated with the overall relative neutron excess $I=$ $(N-Z) / A$ of nuclei. This fact is in agreement with the experimental findings using information from antiprotonic atoms. The DM expression for the neutron skin thickness contains "bulk" and "surface" parts. The bulk part corresponds to the contribution proportional to the distance $t$ between the neutron and proton mean surface locations. This part is quite dependent on the Skyrme force or RMF parametrization used to compute it (see Fig. 2). In finite nuclei, this DM bulk contribution systematically underestimates the neutron skin thickness extracted directly as the difference of the neutron and proton rms radii of the nucleus from self-consistent ETF calculations with effective forces. This evidence indicates that the surface part, due to the $b_{n} \neq b_{p}$ contribution, is necessary in the DM formula to properly estimate the neutron skin thickness in finite nuclei in mean-field models using effective nuclear interactions.

The DM surface contribution $\Delta R_{n p}^{\mathrm{sw}}$ to the neutron skin thickness is smaller than the bulk part and shows a well-defined linear increasing tendency with the overall relative neutron excess $I$. We have investigated the dependence of the slope $\sigma^{\text {sw }}$ with respect to $I$ of this surface contribution using various Skyrme and RMF forces. We have found that the slopes $\sigma^{\text {sw }}$ lie in a region of the $\sigma^{\mathrm{sw}}-J / Q$ plane that can be roughly limited by two straight lines as a function of the $J / Q$ value. It implies that nuclear properties, which are included in the calibration of the free parameters of the Skyrme and RMF interactions, constrain the possible values of the surface width contribution to the neutron skin thickness in the DM. If the same nuclear interaction is used, a good agreement between both the DM formula and the self-consistent ETF calculations of $\Delta R_{n p}$ in finite nuclei is found along the whole periodic table when the contribution $\Delta R_{n p}^{\mathrm{sw}}$ is included in the DM.

To analyze possible bounds suggested by experimental neutron skin data on the value of the $J / Q$ ratio, we have adjusted the DM neutron skin thickness formula to the neutron skin sizes measured in antiprotonic atoms $[48,49]$. We have determined a window $0.6 \lesssim J / Q \lesssim 0.9$ for the $J / Q$ 
ratio by using the largest and smallest surface contributions $\Delta R_{n p}^{\mathrm{sw}}$ obtained from successful Skyrme forces and RMF parametrizations. These two fits reproduce the experimental data with almost the same quality. In other words, the experimental data of the neutron skin thickness in finite nuclei constrain the total theoretical estimate but not its partition into a bulk and a surface contribution. Once the window of $J / Q$ values is known, the compatible range of values of the parameter $L$ can be estimated from the linear correlation between $L$ and $J / Q$ shown in Fig. 1. From our analysis we find the constraints $30 \lesssim L \lesssim 80 \mathrm{MeV}$.

If a model symmetry energy $c_{\text {sym }}(\rho)=J\left(\rho / \rho_{0}\right)^{\gamma}$ is assumed, a prediction for the value of the stiffness $\gamma$ of the symmetry energy can be obtained, with use of the empirical values of $J$ and $\rho_{0}$. In this way we find the estimate $0.32 \lesssim$ $\gamma \lesssim 0.84$. Thus, our analysis of the experimental neutron skins deduced from antiprotonic atoms suggests a relatively soft symmetry energy, in good accord with the recent indications from pygmy [24] and giant [25] dipole resonances. Our prediction for the stiffness $\gamma$ of the symmetry energy also is in reasonable agreement with the constraints derived by Danielewicz [29], by Famiano et al. [20], and by Shetty et al. [19] using different observables, as well as with the value $\gamma=0.51$ of the EOS of the Thomas-Fermi model of Myers and Świątecki $[67,68]$. In the upper limit, our prediction overlaps with the lower limit provided by the analysis of isospin diffusion data in intermediate-energy heavy-ion collisions [12-16].

In summary, different techniques of extracting the parameters that describe the density dependence of the symmetry energy predict different values. However, taking the average of the central values of the predictions displayed in Fig. 6, with account of their uncertainties when available, there exist narrow windows of the parameters $L \sim 45-75 \mathrm{MeV}$ and $\gamma \sim$ $0.5-0.8$ which are, actually, compatible with all the different methods mentioned to obtain them. These ranges of values of the indicated parameters for describing the leading density dependence of the symmetry energy in bulk matter seem to be the "optimal" ones according to present experimental evidence from nuclear data.

To conclude, we are aware that the neutron skin thickness data derived from antiprotonic atoms are to some extent model dependent and have for some nuclei large error bars. Also, our theoretical method represents just an average approximation. In spite of these limitations, we hope to have shown that from neutron skin data it is possible to make a reasonable estimate of the density dependence of the symmetry energy with uncertainties that are not significantly much larger than those currently obtained from other experimental observables. One expects that future data from the planned parity-violating electron scattering experiment for measuring the neutron radius in ${ }^{208} \mathrm{~Pb}$ [2] will contribute to narrow down the constraints derived here from the thickness of neutron skins of nuclei.

\section{ACKNOWLEDGMENTS}

The authors are very grateful to W. J. Świątecki for valuable discussions during the XIV Nuclear Physics Workshop held in Kazimierz-Dolny (Poland). Helpful correspondence with A. Trzcińska is gratefully acknowledged. Work partially supported by Grant Nos. FIS2005-03142 and FIS2008-01661 from MEC (Spain) and FEDER, No. 2009SGR-1289 from Generalitat de Catalunya, and by the Spanish ConsoliderIngenio 2010 Programme CPAN CSD2007-00042. X.R. also was supported by grant AP2005-4751 from MEC (Spain). M.W. gratefully acknowledges the warm hospitality extended to him by the Nuclear Theory Group and the Departament d'Estructura i Constituents de la Matèria at the University of Barcelona during his stay in Barcelona.

\section{APPENDIX}

To compute the surface stiffness coefficient $Q$ and also the neutron and proton surface widths $b_{n}$ and $b_{p}$ that appear in the contribution $\Delta R_{n p}^{\mathrm{sw}}$ of Eq. (10) to the neutron skin thickness, we obtain the self-consistent neutron and proton density profiles in asymmetric semi-infinite nuclear matter (ASINM). To do that we consider a semi-infinite slab with a plane interface separating a mixture of protons and neutrons at the left, whose densities decrease smoothly to zero at the right as empty space is reached. The axis perpendicular to the interface is taken to be the $z$ axis. Thus, the relative neutron excess $\delta=\left(\rho_{n}-\rho_{p}\right) /\left(\rho_{n}+\rho_{p}\right)$ depends locally on the $z$ coordinate. When $z$ goes to minus infinity, the neutron and proton densities approach the values of asymmetric uniform nuclear matter in equilibrium, corresponding to an interior bulk neutron excess $\delta_{0}$.

To obtain the proton and neutron densities in ASINM one has to minimize the total energy per unit area with respect to arbitrary variations of the densities, with the constraint of conservation of the number of protons and neutrons. When $\delta_{0}$ is not very large, so that occurrence of drip nucleons does not take place (which is the situation in all cases considered in the present work), the constrained energy per unit area reads $[53,59,72]$

$$
\frac{E}{S}=\int_{-\infty}^{\infty}\left[\varepsilon(z)-\mu_{n} \rho_{n}(z)-\mu_{p} \rho_{p}(z)\right] d z .
$$

In this equation, $\varepsilon(z)$ represents the energy density functional of the nuclear effective interaction under investigation, and $\mu_{n}$ and $\mu_{p}$ are the neutron and proton chemical potentials. The explicit expressions of $\varepsilon(z)$ in the extended Thomas-Fermi (ETF) method for Skyrme forces and relativistic mean-field interactions can be found in, e.g., Appendix A of Ref. [53]. The proton and neutron densities obey the coupled local EulerLagrange equations

$$
\frac{\delta \varepsilon(z)}{\delta \rho_{n}}-\mu_{n}=0, \quad \frac{\delta \varepsilon(z)}{\delta \rho_{p}}-\mu_{p}=0 .
$$

We solve them fully self-consistently by numerical iteration [54]. We note in this respect that we have not used any parameterized form of the densities such as, e.g., Fermi shapes. In the relativistic model the variational equations (A2) are supplemented with additional field equations for the meson fields; the calculational details for the relativistic problem can be found in Refs. [53-55]. 
From the calculated density profiles, one obtains the mean locations of the surfaces, $z_{0 q}(q=n, p)$, as

$$
z_{o q}=\frac{\int_{-\infty}^{\infty} z \rho_{q}^{\prime}(z) d z}{\int_{-\infty}^{\infty} \rho_{q}^{\prime}(z) d z}
$$

where the primes indicate a derivative with respect to the $z$ coordinate. The proton and neutron surface widths in ASINM are obtained as the second moment of $\rho^{\prime}(z)$ :

$$
b_{q}^{2}=\frac{\int_{-\infty}^{\infty}\left(z-z_{0 q}\right)^{2} \rho_{q}^{\prime}(z) d z}{\int_{-\infty}^{\infty} \rho_{q}^{\prime}(z) d z} .
$$

The distance $t=z_{0 n}-z_{0 p}$ between the mean surface locations of the neutron and proton density profiles allows one to extract the surface stiffness coefficient $Q$ from the relation [56]

$$
t=z_{0 n}-z_{0 p}=\frac{3 r_{0}}{2} \frac{J}{Q} \delta_{0},
$$

which is valid in the limit of small asymmetries. For each given nuclear interaction we solve the ASINM problem for five different values of $\delta_{0}$, between 0.005 and 0.025 , and we then evaluate $Q$ from the slope of $t$.
There exists a second way of computing $Q$. It is based on the fact that in dividing the energy in bulk and surface parts, as soon as $\delta_{0} \neq 0$, there are two possibilities to define the bulk reference energy $[53,72]$. One definition is based on the chemical potentials $\mu_{n}$ and $\mu_{p}$ of each nucleon species; this definition is thermodynamically consistent and it is the one that we have given in Eq. (A1). The second definition of the reference energy is based on taking the value of the energy per particle in bulk asymmetric nuclear matter. Accordingly, on the bulk reference energy chosen, there exist two forms of the surface energy in ASINM, which are called $E_{\text {surf }, \mu}$ and $E_{\text {surf,e }}[53,72]$. In the small asymmetry limit, it can be shown that the difference between these two quantities behaves as

$$
E_{\text {surf,e }}-E_{\text {surf }, \mu}=\frac{9 J^{2}}{2 Q} \delta_{0}^{2} .
$$

Thus, the slope of $E_{\text {surf,e }}-E_{\text {surf }, \mu}$ with respect to $\delta_{0}^{2}$ provides another means to extract the value of the surface stiffness coefficient $Q$. We have computed $Q$ from Eq. (A5) and have used Eq. (A6) to confirm the validity of our calculated values.
[1] I. Angeli, At. Data Nucl. Data Tables 87, 185 (2004).

[2] R. Michaels, P. A. Souder, and G. M. Urciuoli, Jefferson Laboratory Experiment E06002, http://hallaweb.jlab.org/parity/prex.

[3] J. M. Lattimer and M. Prakash, Science 304, 536 (2004); Phys. Rep. 442, 109 (2007).

[4] H. Heiselberg and M. Hjorth-Jensen, Phys. Rep. 328, 237 (2000).

[5] A. W. Steiner, M. Prakash, J. M. Lattimer, and P. J. Ellis, Phys. Rep. 411, 325 (2005).

[6] T. Sil, M. Centelles, X. Viñas, and J. Piekarewicz, Phys. Rev. C 71, 045502 (2005), and references therein.

[7] P. Jofré, A. Reisenegger, and R. Fernández, Phys. Rev. Lett. 97, 131102 (2006); P. G. Krastev and Bao-An Li, Phys. Rev. C 76, 055804 (2007).

[8] Dao T. Khoa, W. von Oertzen, and A. A. Ogloblin, Nucl. Phys. A602, 98 (1996).

[9] T. Gaitanos, M. Di Toro, S. Typel, V. Baran, C. Fuchs, V. Greco, and H. H. Wolter, Nucl. Phys. A732, 24 (2004).

[10] V. Baran, M. Colonna, V. Greco, and M. Di Toro, Phys. Rep. 410, 335 (2005).

[11] M. B. Tsang et al., Phys. Rev. Lett. 92, 062701 (2004).

[12] A. W. Steiner and B. A. Li, Phys. Rev. C 72, 041601(R) (2005).

[13] L. W. Chen, C. M. Ko, and B. A. Li, Phys. Rev. Lett. 94, 032701 (2005).

[14] L. W. Chen, C. M. Ko, and B. A. Li, Phys. Rev. C 72, 064309 (2005).

[15] L. W. Chen, C. M. Ko, and B. A. Li, Phys. Rev. C 76, 054316 (2007).

[16] B. A. Li, L. W. Chen, and C. M. Ko, Phys. Rep. 464, 113 (2008).

[17] G. A. Souliotis, D. V. Shetty, A. Keksis, E. Bell, M. Jandel, M. Veselsky, and S. J. Yennello, Phys. Rev. C 73, 024606 (2006).

[18] D. V. Shetty, S. J. Yennello, and G. A. Souliotis, Phys. Rev. C 75, 034602 (2007).

[19] D. V. Shetty, S. J. Yennello, and G. A. Souliotis, Phys. Rev. C 76, 024606 (2007).
[20] M. A. Famiano et al., Phys. Rev. Lett. 97, 052701 (2006).

[21] M. B. Tsang, Y. Zhang, P. Danielewicz, M. Famiano, Z. Li, W. G. Lynch, and A. W. Steiner, Phys. Rev. Lett. 102, 122701 (2009).

[22] J. Piekarewicz, Phys. Rev. C 66, 034305 (2002); 69, 041301(R) (2004).

[23] T. Li et al., Phys. Rev. Lett. 99, 162503 (2007).

[24] A. Klimkiewicz et al., Phys. Rev. C 76, 051603(R) (2007).

[25] L. Trippa, G. Colò, and E. Vigezzi, Phys. Rev. C 77, 061304(R) (2008).

[26] H. Liang, N. Van Giai, and J. Meng, Phys. Rev. Lett. 101, 122502 (2008).

[27] W. D. Myers and W. J. Świątecki, Nucl. Phys. A336, 267 (1980).

[28] W. J. Świątecki, A. Trzcińska, and J. Jastrzębski, Phys. Rev. C 71, 047301 (2005).

[29] P. Danielewicz, Nucl. Phys. A727, 233 (2003).

[30] P. Danielewicz and J. Lee, Nucl. Phys. A818, 36 (2009).

[31] C. J. Pethick and D. G. Ravenhall, Nucl. Phys. A606, 173 (1996).

[32] S. Mizutori, J. Dobaczewski, G. A. Lalazissis, W. Nazarewicz, and P.-G. Reinhard, Phys. Rev. C 61, 044326 (2000).

[33] R. J. Furnstahl, Nucl. Phys. A706, 85 (2002).

[34] J. Treiner and H. Krivine, Ann. Phys. 170, 406 (1986).

[35] M. Warda, B. Nerlo-Pomorska, and K. Pomorski, Nucl. Phys. A635, 484 (1998).

[36] A. E. L. Dieperink, Y. Dewulf, D. Van Neck, M. Waroquier, and V. Rodin, Phys. Rev. C 68, 064307 (2003).

[37] B. A. Brown, Phys. Rev. Lett. 85, 5296 (2000).

[38] S. Typel and B. A. Brown, Phys. Rev. C 64, 027302 (2001).

[39] M. Centelles, M. Del Estal, X. Viñas, and S. K. Patra, in The Nuclear Many-Body Problem 2001, Vol. 53 of NATO Advanced Studies Institute Series B: Physics, edited by W. Nazarewicz and D. Vretenar (Kluwer, Dordrecht, 2002), p. 97.

[40] M. Baldo, C. Maieron, P. Schuck, and X. Viñas, Nucl. Phys. A736, 241 (2004).

[41] S. S. Avancini, J. R. Marinelli, D. P. Menezes, M. M. W. Moraes, and C. Providência, Phys. Rev. C 75, 055805 (2007). 
[42] L. Ray, G. W. Hoffmann, G. S. Blanpied, W. R. Coker, and R. P. Liljestrand, Phys. Rev. C 18, 1756 (1978); L. Ray and G. W. Hoffmann, Phys. Rev. C 31, 538 (1985).

[43] V. E. Starodubsky and N. M. Hintz, Phys. Rev. C 49, 2118 (1994).

[44] S. Karataglidis, K. Amos, B. A. Brown, and P. K. Deb, Phys. Rev. C 65, 044306 (2002).

[45] B. C. Clark, L. J. Kerr, and S. Hama, Phys. Rev. C 67, 054605 (2003).

[46] A. Krasznahorkay et al., Phys. Rev. Lett. 82, 3216 (1999).

[47] A. Krasznahorkay et al., Nucl. Phys. A731, 224 (2004).

[48] A. Trzcińska, J. Jastrzębski, P. Lubiński, F. J. Hartmann, R. Schmidt, T. von Egidy, and B. Kłos, Phys. Rev. Lett. 87, 082501 (2001).

[49] J. Jastrzębski, A. Trzcińska, P. Lubiński, B. Kłos, F. J. Hartmann, T. von Egidy, and S. Wycech, Int. J. Mod. Phys. E 13, 343 (2004).

[50] B. Kłos et al., Phys. Rev. C 76, 014311 (2007).

[51] M. Centelles, X. Roca-Maza, X. Viñas, and M. Warda, Phys. Rev. Lett. 102, 122502 (2009).

[52] M. Brack, C. Guet, and H.-B. Håkansson, Phys. Rep. 123, 275 (1985).

[53] M. Centelles, M. Del Estal, and X. Viñas, Nucl. Phys. A635, 193 (1998).

[54] M. Centelles, M. Pi, X. Viñas, F. Garcias, and M. Barranco, Nucl. Phys. A510, 397 (1990).

[55] M. Centelles, X. Viñas, M. Barranco, and P. Schuck, Ann. Phys. (NY) 221, 165 (1993).
[56] W. D. Myers and W. J. Świątecki, Ann. Phys. (NY) 55, 395 (1969); 84, 186 (1974).

[57] W. D. Myers, Droplet Model of Atomic Nuclei (Plenum, New York, 1977).

[58] M. Farine, J. M. Pearson, and B. Rouben, Nucl. Phys. A304, 317 (1978); M. Farine, J. Côté, and J. M. Pearson, ibid. A338, 86 (1980); Phys. Rev. C 24, 303 (1981).

[59] K. Kolehmainen, M. Prakash, J. M. Lattimer, and J. Treiner, Nucl. Phys. 439, 535 (1985).

[60] M. Centelles and X. Viñas, Nucl. Phys. A563, 173 (1993).

[61] J. Piekarewicz and M. Centelles, Phys. Rev. C 79, 054311 (2009).

[62] M. Lopez-Quelle, S. Marcos, R. Niembro, A. Bouyssy, and Nguyen Van Giai, Nucl. Phys. A483, 479 (1988).

[63] I. Bednarek and R. Manka, J. Phys. G 36, 095201 (2009).

[64] S. Kowalski et al., Phys. Rev. C 75, 014601 (2007).

[65] S. K. Samaddar, J. N. De, X. Viñas, and M. Centelles, Phys. Rev. C 76, 041602(R) (2007); 78, 034607 (2008).

[66] S. R. Souza, M. B. Tsang, R. Donangelo, W. G. Lynch, and A. W. Steiner, Phys. Rev. C 78, 014605 (2008).

[67] W. D. Myers and W. J. Świątecki, Nucl. Phys. A601, 141 (1996).

[68] W. D. Myers and W. J. Świątecki, Phys. Rev. C 57, 3020 (1998).

[69] A. Schwenk and C. J. Pethick, Phys. Rev. Lett. 95, 160401 (2005).

[70] J. Piekarewicz, Phys. Rev. C 76, 064310 (2007).

[71] Z. Xiao, B. A. Li, L. W. Chen, G. C. Yong, and M. Zhang, Phys. Rev. Lett. 102, 062502 (2009).

[72] W. D. Myers, W. J. Świątecki, and C. S. Wong, Nucl. Phys. A436, 185 (1985). 